\title{
Laparoscopic nephrectomy
}

\author{
Kurt Kerbl, Ralph V Clayman, Elspeth M McDougall, Louis R Kavoussi
}

This is the fourth in a series of articles describing current techniques in minimal access surgery. The articles have been written to inform non-specialists of development in this rapidly moving subject.

Department of Surgery

(Division of Urology), Washington University School of Medicine, 4960 Children's Place, St Louis, MO 63110, USA

Kurt Kerbl, fellow

Ralph V Clayman, professor of urologic surgery and radiology

Elspeth M McDougall, assistant professor of urology

Department of Urology, Johns Hopkins University, Baltimore, MD 21224, USA Louis R Kavoussi, associate professor of urology

Correspondence to: Professor Clayman

$B M \mp 1993 ; 307: 1488-9$

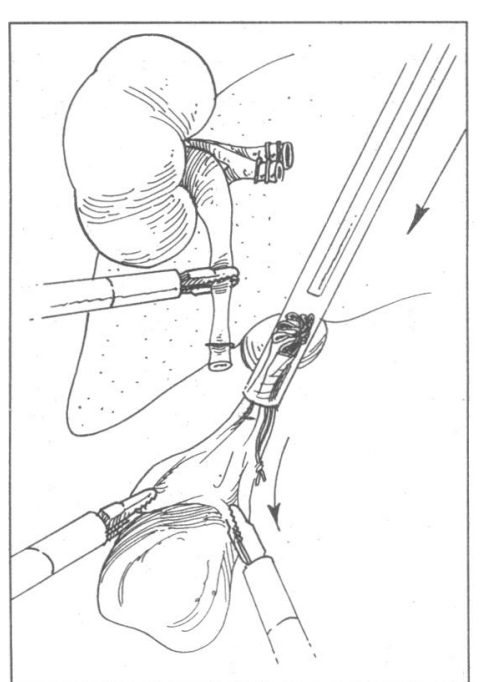

FIG 1-Organ entrapment sack passed into abdomen and unfolded

\begin{abstract}
Since the first clinical operation in June 1990 laparoscopic nephrectomy for benign renal disease has become widely accepted. Although the laparoscopic operation takes much longer than open surgery, there are considerable reductions in the length of postoperative hospital stay and the time taken to return to normal activities and to full recovery. Major complications were relatively common in early operations, but with more experience morbidity has been reduced. Laparoscopic nephrectomy for malignant renal disease is still controversial, largely because of the fear of release of malignant tissue into the abdominal cavity during the morcellation and retrieval of the diseased kidney. To prevent this, the kidney is removed intact through a $5-7 \mathrm{~cm}$ incision. Long term follow up is needed, however, before we will know whether the laparoscopic procedure is effective in preventing recurrence of cancer. New developments have improved various technical aspects of the operation, but stringent assessment of new techniques is necessary so that the medical community can decide which procedures should become routine practice.
\end{abstract}

Percutaneous removal of the kidney began in 1988 when Smith and coworkers attempted to remove a pig kidney by means of a single tract, percutaneous retroperitoneal approach. ${ }^{1}$ Their sole laboratory experience was complicated by a colonic injury, and the project was abandoned. Subsequently, Ikari et al reported a single clinical case in which they attempted to remove a non-functional kidney by a single tract, percutaneous retroperitoneal approach. ${ }^{2}$ After first embolising the renal artery, they used myriad grasping forceps to avulse bits of renal parenchymal tissue, which were then delivered through the nephrostomy tract. A total of $16 \mathrm{~g}$ of tissue was removed, but postoperative sonography of the flank showed that about $17 \mathrm{~g}$ of kidney tissue remained in place. The advent of laparoscopic techniques stimulated us to reexamine feasibility of developing a minimally invasive approach to nephrectomy. ${ }^{3}$ Using a laparoscopic

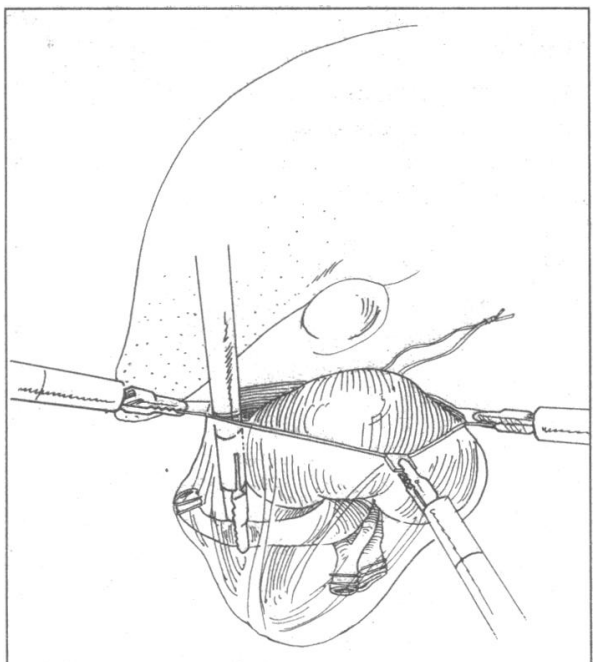

FIG 2-Dissected kidney placed in entrapment sack approach, we completely dissected a pig kidney and secured the renal vessels and ureter with laparoscopic staples. We then passed a newly designed organ entrapment sack (Cook Urological, Spencer, IN) into the abdomen and manoeuvred the kidney into the sack. The neck of the sack was brought to the surface of the abdomen via the $12 \mathrm{~mm}$ port entry site at the umbilicus. The kidney was morcellated and the fragments aspirated with a recently developed high speed electrical tissue morcellator (Cook Urological). The empty sack was then pulled from the abdomen.

Our laboratory experience was subsequently expanded into the clinical realm. Using five entry ports, we successfully performed the first clinical laparoscopic nephrectomy at Barnes Hospital, Washington University, in June 1990. Since then 30 laparoscopic nephrectomies for benign renal disease have been performed at our institution and more than 100 have been performed at other centres worldwide. ${ }^{4-9}$ We recently compared the first 20 of these operations with a contemporary series of 23 open surgical nephrectomies performed for benign renal disease and a contemporary series of 29 patients who underwent donor nephrectomies (the healthiest subgroup of all patients in whom nephrectomy is performed). The laparoscopic operations took much longer (355 minutes) than the open procedures, but our patients showed considerably reduced times for their postoperative hospital stay (three to four days), return to normal activities (one month), and full recovery (less than two months). As with all laparoscopic procedures, however, there is a pronounced learning curve for the operation. In our first 26 laparoscopic nephrectomies for benign disease there were no mortalities, but three $(12 \%)$ of the patients suffered major morbidity and five $(19 \%)$ suffered minor morbidity. The major complications were conversion to an open procedure because of the inaccessibility of the renal artery in a morbidly obese patient, a right brachial plexus palsy due to positioning (this resolved over 12 months), a pulmonary embolism, and a pneumothorax. The occurrence of the major complications reflected our experience: three occurred No in our first 12 patients and only one in our next 12 patients.

\section{Treatment of malignant disease}

While laparoscopic nephrectomy for benign disease has been widely accepted by surgeons, laparoscopic nephroureterectomy and nephrectomy for malignant disease remain controversial. The main concern is the potential for tumour seeding at the time of tissue morcellation and organ retrieval. The current approach is therefore to entrap and remove the diseased kidney intact via a $5-7 \mathrm{~cm}$ incision. This means that seeding is no longer an issue and histological analysis of the kidney can be made to accurately measure the stage and grade of the tumour.

The first laparoscopic nephroureterectomy for transitional cell cancer was performed in May 1991. The procedure was carried out like other laparoscopic nephrectomies but with the additional dissection of the entire ureter and a cuff of bladder, which was secured 
FIG 3-Neck of sack is pulled to surface of abdomen, and kidney is morcellated and aspirated by high speed morcellator

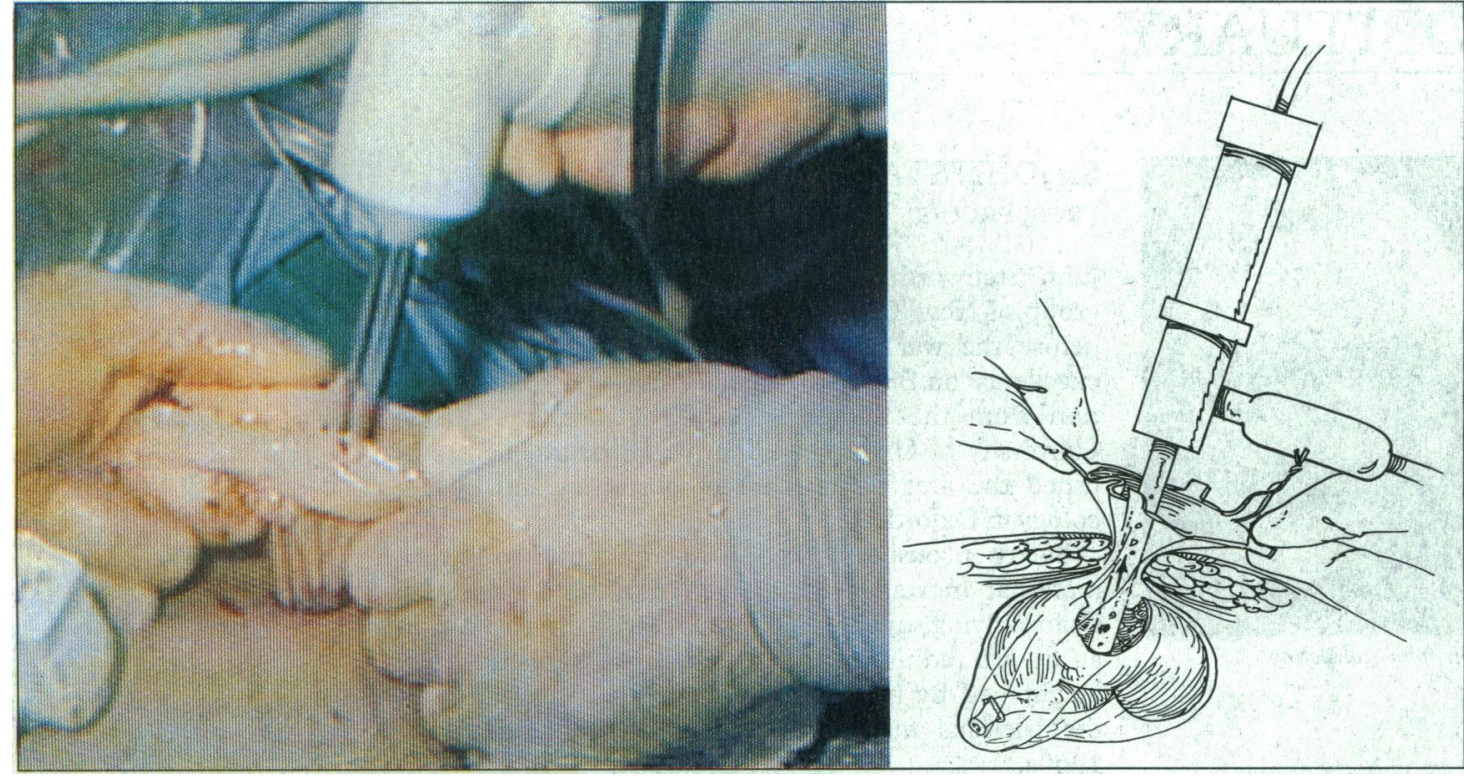

and incised with a $12 \mathrm{~mm}$ laparoscopic tissue stapler. ${ }^{10}$ Since then seven patients have undergone laparoscopic nephroureterectomy for transitional cell cancer of the upper urinary tract at our institution. A comparison of our first six laparoscopic nephroureterectomies with a contemporary series of eight open nephroureterectomies revealed that the operative time for laparoscopic nephroureterectomy was 2.5 times longer than for open surgery but the laparoscopic patients had a shorter hospital stay ( 4.6 days $v 9 \cdot 25$ days), requested less parenteral analgesia (24 mg $v 149 \mathrm{mg}$ morphine sulphate equivalent), returned to usual activities more rapidly ( 2.6 weeks $v 6$ weeks), and were fully recovered much earlier ( 5 weeks $v 7.5$ months).

Laparoscopic nephrectomy has also been performed for primary renal parenchymal tumours. Two of us (LRK and RVC) recently examined the results of their first eight laparoscopic radical or total nephrectomies for renal tumours smaller than $6 \mathrm{~cm}$ in diameter. For three patients with upper pole tumours the ipsilateral adrenal gland was excised together with the specimen. The mean operating time was seven hours, but these patients left the hospital in four days, returned to their usual activities in two weeks, and were fully recovered in four weeks. In comparison, for a contemporary series of 33 patients undergoing open radical nephrectomy for renal tumours smaller than $6 \mathrm{~cm}$ the hospital stay was 7.5 days, return to work occurred after $7 \cdot 1$ weeks, and complete convalescence required $6 \cdot 8$ months (LRK, personal communication).

Assessments-The laparoscopic approach to malignant renal disease will require much longer follow up and careful analysis before its role can properly be

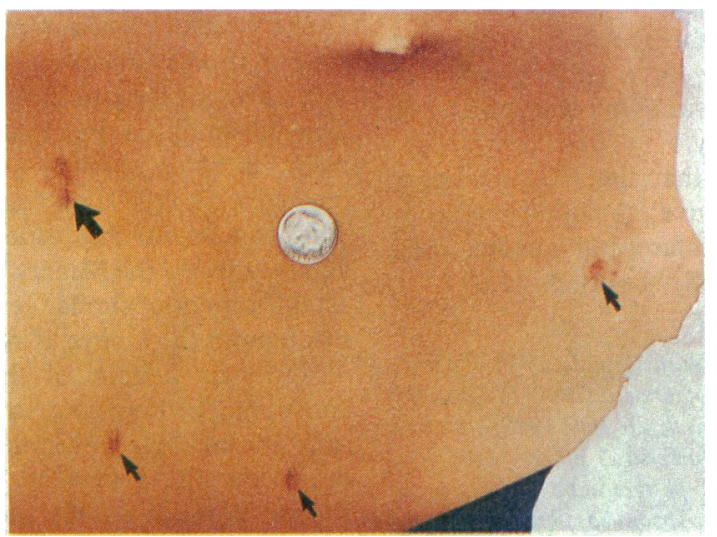

FIG 4-At two weeks after laparoscopic nephrectomy only small scars visible no larger than a dime (size of $5 p$ piece) determined. The immediate benefits of shorter hospital stay and convalescence are relatively unimportant compared with the ultimate cure of the cancer, which is the final determinant of the efficacy of the procedure.

\section{New developments}

Laparoscopic nephrectomy is still being developed, and several investigators have refined and improved various technical aspects. Initially, almost all laparoscopic nephrectomies were performed with a transperitoneal approach, but Gaur's recent development of a balloon device for dilatation of the retroperitoneum has allowed urologists direct retroperitoneal access. ${ }^{11}$ This approach facilitates the initial renal dissection as the intra-abdominal organs no longer need to be mobilised in order to uncover the renal fossa. ${ }^{12}$

The fast and widespread adoption of laparoscopic techniques is currently being spurred on by enthusiastic doctors, extensive and favourable press coverage, and a lucrative and growing market in laparoscopic instrumentation. New laparoscopic procedures are being described almost on a weekly basis, but careful critical comparison of each newly developed procedure with its counterpart in open surgery is essential. Only in this manner will the medical community be able to decide which of the new procedures should become routine practice.

1 Weinberg J, Smith $\mathrm{AD}$. Percutaneous resection of the kidney: preliminary report. Fournal of Endourology 1988;2:355-61.

2 Ikari O, Netto NT Jr, Palma PCR, D'Ancona CA. Percutaneous nephrectomy in nonfunctioning kidneys: a preliminary report. $₹$ Urol 1990;144:966-8.

3 Clayman RV, Kavoussi LR, Long SL, Dierks SM, Meretyk S, Soper NJ. Laparoscopic nephrectomy: initial report of pelviscopic organ ablation in the pig. Foumal of Endourology 1990;4:247-52.

4 Rassweiler J, Potempa DM, Henkel TO, Guenther M, Tschada R, Alken P. The technical aspects of transperitoneal laparoscopic nephrectomy (TLN), adrenalectomy (TLA) and nephroureterectomy (abstract). fournal of Endourology 1992;6:S58.

5 Coptcoat MJ, Joyce AJ, Eden C, Rassweiler J. Laparoscopic nephrectomythe King's experience. Minimally Invasive Therapy 1992;1 (suppl 1):B25.

6 Smith AD, Badlani GH, Weiss GH. The lateral oblique position for laparoscopic nephrectomy. Minimally Invasive Therapy 1992;1 (suppl 1):B21.

7 Ehrlich RM, Gershman A, Fuchs G. Laparoscopic nephrectomy in a child: expanding horizons for laparoscopy in pediatric urology. Foumal of Endo-
entich RM, Gersman A, Fuchs G. Laparoscopic nephrectomy in a child: expanding horizons

8 Gaur DD, Agarwal DK, Purohit KC. Retroperitoneal laparoscopic nephrectomy: initial case report. $\{$ Urol 1993;149:103-5.

9 Matsuda T, Uchida J, Kawamura H, Komatz Y, Fujise K, Terachi T, et al. [Experience with laparoscopic nephrectomy]. Hinyokika Kiyo 1992;38(7): 759-65.

0 Kerbl K, Chandhoke PS, McDougall E, Figenshau RS, Stone AM, Clayman RV. Laparoscopic stapled bladder closure: laboratory and clinical experience. 7 Urol 1993;149:1437-40.

11 Gaur DD. Laparoscopic operative retroperitoneoscopy: use of a new device. I Urol 1992;148:1137-9.

12 Kerbl K, Figenshau RS, Clayman RV, Chandhoke PS, Kavoussi LR, Albala DM et al. Retroperitoneal laparoscopic nephrectomy: laboratory and clinical experience. Foumal of Endourology 1993;7:23-6. 